Posi tron accel er at i on to ul trarel at i vi st i c energi es by an obl i que nagnet osoni c shock wave i $n$ an el ectron- posi tron- $\mathrm{i}$ on pl asma

\begin{tabular}{|l|l|}
\hline $\begin{array}{l}\text { j our nal or } \\
\text { publ i cat i on } \mathrm{t} \text { i t l e }\end{array}$ & Physi cs of Pl asmas \\
\hline vol une & Vol . 12 \\
\hline number & I ssue1 \\
\hline page r ange & $012312-1-012312-7$ \\
\hline year & 2004 12-01 \\
\hline URL & ht t p: //hdl . handl e. net /10655/8962 \\
\hline
\end{tabular}

doi: $10.1063 / 1.1827624$ 


\section{AIP Plasmas}

\section{Positron acceleration to ultrarelativistic energies by an oblique magnetosonic shock wave in an electron-positron-ion plasma}

Hiroki Hasegawa and Yukiharu Ohsawa

Citation: Phys. Plasmas 12, 012312 (2005); doi: 10.1063/1.1827624

View online: http://dx.doi.org/10.1063/1.1827624

View Table of Contents: http://pop.aip.org/resource/1/PHPAEN/v12/i1

Published by the American Institute of Physics.

\section{Related Articles}

Test particle simulation of direct laser acceleration in a density-modulated plasma waveguide Phys. Plasmas 19, 113104 (2012)

Ionization effects in the generation of wake-fields by ultra-high contrast femtosecond laser pulses in argon gas Phys. Plasmas 19, 103104 (2012)

Ion acceleration from thin foil and extended plasma targets by slow electromagnetic wave and related ion-ion beam instability

Phys. Plasmas 19, 103105 (2012)

Observation of a strong correlation between electromagnetic soliton formation and relativistic self-focusing for ultra-short laser pulses propagating through an under-dense plasma

Phys. Plasmas 19, 102304 (2012)

Hot-electron production and suprathermal heat flux scaling with laser intensity from the two-plasmon-decay instability

Phys. Plasmas 19, 102703 (2012)

\section{Additional information on Phys. Plasmas}

Journal Homepage: http://pop.aip.org/

Journal Information: http://pop.aip.org/about/about_the_journal

Top downloads: http://pop.aip.org/features/most_downloaded

Information for Authors: http://pop.aip.org/authors

\section{ADVERTISEMENT}

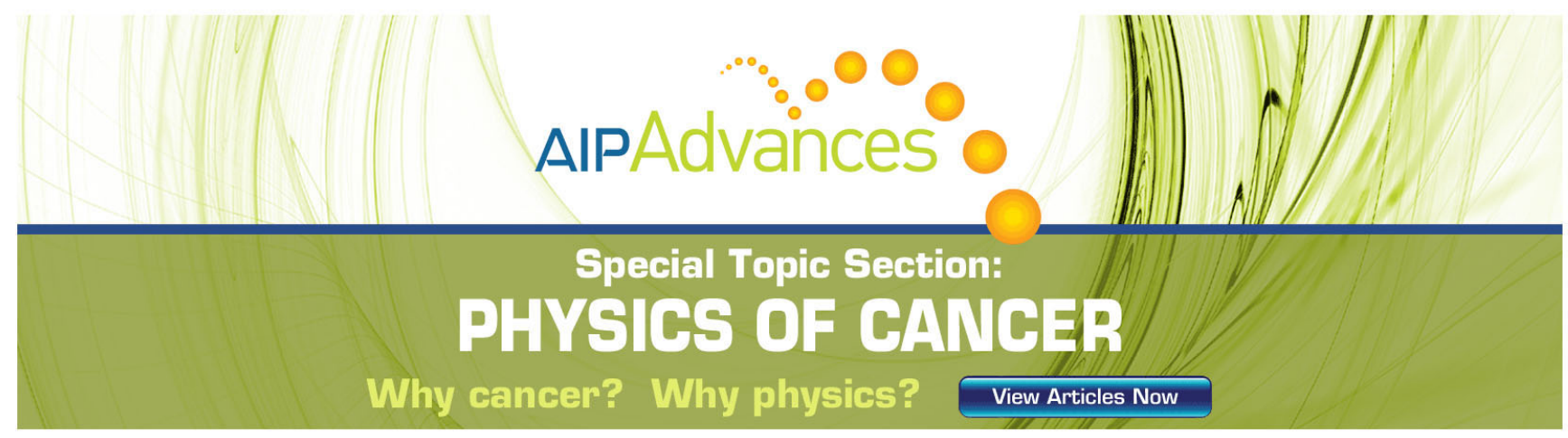




\title{
Positron acceleration to ultrarelativistic energies by an oblique magnetosonic shock wave in an electron-positron-ion plasma
}

\author{
Hiroki Hasegawa \\ Theory and Computer Simulation Center, National Institute for Fusion Science, Toki 509-5292, Japan \\ Yukiharu Ohsawa ${ }^{\text {a) }}$ \\ Department of Physics, Nagoya University, Nagoya 464-8602, Japan
}

(Received 16 June 2004; accepted 8 October 2004; published online 14 December 2004)

\begin{abstract}
Positron acceleration in a shock wave in a plasma consisting of electrons, positrons, and ions is studied with theory and simulations. From the relativistic equation of motion, it is found that an oblique shock wave can accelerate some positrons with the energy increase rate proportional to $\mathbf{E} \cdot \mathbf{B}$. They move nearly parallel to the external magnetic field, staying in the shock transition region for long periods of time. Then, this acceleration is demonstrated with one-dimensional, relativistic, electromagnetic particle simulations with full particle dynamics. Some positrons have been accelerated to ultrarelativistic energies $(\gamma \sim 1000)$ with this mechanism. Parametric study of this acceleration is also made. (C) 2005 American Institute of Physics. [DOI: 10.1063/1.1827624]
\end{abstract}

\section{INTRODUCTION}

Plasmas containing positrons can be produced with intense lasers ${ }^{1}$ and could be found around pulsars or in astrophysical jets. ${ }^{2-5}$ Many authors have thus studied wave propagation, plasma configuration, particle acceleration, etc., in such plasmas. ${ }^{6-18}$

In a recent paper, ${ }^{19}$ it has been shown that an oblique magnetosonic shock wave in an electron-positron-ion $(e-p-i)$ plasma can accelerate positrons to ultrarelativistic energies. During the acceleration, these positrons stay in the shock transition region and move nearly parallel to the external magnetic field $\mathbf{B}_{0}$. The energy increase rate was found to be proportional to $\mathbf{E} \cdot \mathbf{B}$.

These features are quite different from those in the surfatron acceleration, ${ }^{20-23}$ where the longitudinal electric field $E_{x}$ accelerates particles in the direction parallel to the wave front and perpendicular to the magnetic field. For the unlimited acceleration, $E_{x} / B>1$ is required. ${ }^{22}$

This paper describes the positron acceleration found in Ref. 19 in more detail. In Sec. II, we outline some properties of oblique magnetohydrodynamic waves in cold $e-p-i$ plasmas. Linear dispersion relations of the three modes in the frequency regime lower than the electron gyrofrequency are shown. Then, some nonlinear properties of the magnetosonic mode, which is one of the three modes, are mentioned. Although the magnetosonic wave has no electric potential in a pure electron-positron $(e-p)$ plasma, ${ }^{8,12,17}$ it can have large electric potential in an $e-p-i$ plasma, which plays an important role in the particle acceleration.

In Sec. III, we theoretically discuss the positron acceleration. Some positrons are reflected along the magnetic field by $E_{\|}$, the electric field parallel to $\mathbf{B}$. Assuming that $v d \gamma / d t \gg \gamma|d \mathbf{v} / d t|$ for the reflected positrons, where $\mathbf{v}$ is the particle velocity and $\gamma$ is the Lorentz factor, we obtain $\mathbf{v}$ and $d \gamma / d t$ of relativistic positrons. In this solution, $\mathbf{v}$ is nearly

${ }^{a)}$ Electronic mail: ohsawa@phys.nagoya-u.ac.jp parallel to the external magnetic field $\mathbf{B}_{0}$, and $\gamma$ increases linearly with time.

In Sec. IV, we study the positron acceleration by using a one-dimensional (one space coordinate and three velocity components), relativistic, electromagnetic particle simulation code with full particle dynamics. It is shown that some positrons are reflected and then accelerated in the shock transition region. The observed maximum energy in the simulations is $\gamma \sim 1000$. The energy increase rate $d \gamma / d t$ is in good agreement with the theoretical prediction. Also, as the theory predicts, the particle motion is nearly parallel to the external magnetic field. We then examine the dependence of this acceleration on some plasma parameters; shock speed, propagation angle, ion-to-electron mass ratio, and positron density. In Sec. V, we summarize our work.

\section{MAGNETOHYDRODYNAMIC WAVES IN AN $e-p-i$ PLASMA}

Here, we briefly describe magnetohydrodynamic waves in $e-p-i$ plasmas. From the three-fluid model for a cold $e-p-i$ plasma with full Maxwell equations, we obtain linear dispersion relations of oblique waves as

$$
\begin{aligned}
\left(1-\sum_{j} \frac{\omega_{p j}^{2}}{\omega^{2}-\Omega_{j}^{2}}-\frac{c^{2} k^{2}}{\omega^{2}}\right)\left[( 1 - \sum _ { j } \frac { \omega _ { p j } ^ { 2 } } { \omega ^ { 2 } } ) \left(1-\sum_{j} \frac{\omega_{p j}^{2}}{\omega^{2}-\Omega_{j}^{2}}\right.\right. \\
\left.\left.-\frac{c^{2} k^{2}}{\omega^{2}}\right)+\left(\sum_{j} \frac{\omega_{p j}^{2}}{\Omega_{j}^{2}} \frac{\left.\omega^{2}-\Omega_{j}^{2}\right)}{\omega^{2}}\right) \frac{c^{2}}{\omega^{2}} \sin ^{2} \theta\right] \\
-\left(\sum_{j} \frac{\omega_{p j}^{2}}{\left(\omega^{2}-\Omega_{j}^{2}\right)} \frac{\Omega_{j}}{\omega}\right)^{2}\left(1-\sum_{j} \frac{\omega_{p j}^{2}}{\omega^{2}}-\frac{c^{2} k^{2}}{\omega^{2}} \sin ^{2} \theta\right)=0,
\end{aligned}
$$

where $\omega_{p j}$ is the plasma frequency, 


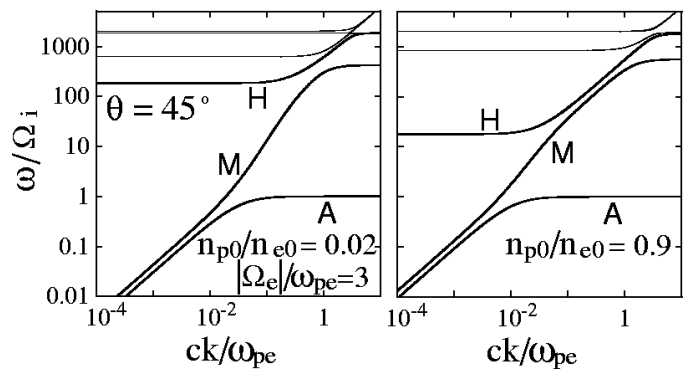

FIG. 1. Dispersion relations for magnetohydrodynamic waves in $e-p-i$ plasmas. The propagation angle is $\theta=45^{\circ}$. The positron densities are $n_{p 0} / n_{e 0}$ $=0.02$ and $n_{p 0} / n_{e 0}=0.9$ in the left and right panels, respectively. In the low frequency regime, we have three modes: the high-frequency mode (line $H$ ), magnetosonic mode (line $M$ ), and Alfvén mode (line $A$ ).

$$
\omega_{p j}^{2}=4 \pi n_{j 0} q_{j}^{2} / m_{j},
$$

with the subscript $j=e$ (electrons), $p$ (positrons), or $i$ (ions), and $\Omega_{j}$ is the gyrofrequency including the sign of the charge,

$$
\Omega_{j}=q_{j} B_{0} /\left(m_{j} c\right) \text {. }
$$

The subscript 0 refers to equilibrium quantities. Equation (1) gives six oblique waves. These are shown in Fig. 1, where the ion mass and charge were taken to be $m_{i} / m_{e}=1836$ and $q_{i}=e$. The magnetic field strength is $\left|\Omega_{e}\right| / \omega_{p e}=3.0$. The angle between the wave vector $\mathbf{k}$ and the external magnetic field $\mathbf{B}_{0}$ is $\theta=45^{\circ}$. The positron-to-electron density ratio is $n_{p 0} / n_{e 0}=0.02$ for the left panel, while it is $n_{p 0} / n_{e 0}=0.9$ for the right panel. These pictures indicate that in the low frequency regime such that $\omega \lesssim\left|\Omega_{e}\right|$, we have three modes. We will call them high-frequency mode (line $H$ ), magnetosonic mode (line $M$ ), and Alfvén mode (line $A$ ), respectively. ${ }^{17-19}$

In the limit of $k \rightarrow \infty$, these waves have resonance frequencies:

$$
\omega_{\mathrm{hfr}}=\left|\Omega_{e}\right|,
$$

for the high-frequency mode,

$$
\omega_{\mathrm{mr}} \approx\left|\Omega_{e}\right| \cos \theta
$$

for the magnetosonic mode, and

$$
\omega_{\mathrm{Ar}} \approx \Omega_{i},
$$

for the Alfvén mode. At $k=0$, the high-frequency mode has a cutoff frequency,

$$
\omega_{\mathrm{hf0}} \approx Z(\mu+\nu)\left|\Omega_{e}\right| /\left(1+Z^{2} \mu \nu\right),
$$

where $\nu=n_{i 0} /\left(n_{e 0}+n_{p 0}\right), \mu=m_{e} / m_{i}$, and $Z=q_{i} / e$.

In the long-wavelength region, the dispersion relation of the magnetosonic wave is given as

$$
\omega=\frac{v_{\mathrm{A}}}{\left(1+v_{\mathrm{A}}^{2} / c^{2}\right)^{1 / 2}}\left(k+\eta_{\mathrm{m}} k^{3}\right),
$$

where $v_{\mathrm{A}}$ is the Alfvén speed

$$
v_{\mathrm{A}}=B_{0}\left(4 \pi \sum_{j} n_{j 0} m_{j}\right)^{-1 / 2}
$$

The dispersion coefficient $\eta_{\mathrm{m}}$ is obtained as

$$
\begin{aligned}
\eta_{\mathrm{m}}= & -\frac{v_{\mathrm{A}}^{4}}{2 c^{2}\left(1+v_{\mathrm{A}}^{2} / c^{2}\right)^{2}}\left[\sum_{j} \frac{\omega_{p j}^{2}}{\Omega_{j}^{4}}\right. \\
& \left.-\frac{v_{\mathrm{A}}^{2}}{\left(c^{2}+v_{\mathrm{A}}^{2}\right) \sin ^{2} \theta}\left(\sum_{j} \frac{\omega_{p j}^{2}}{\Omega_{j}^{3}}\right)^{2}\right] .
\end{aligned}
$$

As expected from Eq. (8), nonlinear magnetosonic waves are governed by the Korteweg-de Vries equation. ${ }^{18}$ The soliton width is $\sim c / \omega_{p i}$ except for the vicinity of $\theta=90^{\circ}$.

The magnetosonic wave has no electric potential in a pure $e-p$ plasma. ${ }^{8,12,17}$ In an $e-p-i$ plasma, however, it can have a large electric potential. The magnitude of the potential in a solitary wave ${ }^{18}$ is given as

$$
e \phi=\frac{2 m_{i} v_{\mathrm{A}}^{2}}{\left(1+v_{\mathrm{A}}^{2} / c^{2}\right)} \frac{\left(1-Z^{2} \mu^{2}\right) \nu}{Z(\mu+\nu)} \frac{(M-1)}{\sin ^{2} \theta},
$$

where $M$ is the Alfvén Mach number. For $n_{i 0} / n_{e 0} \sim 10^{-2}$, the potential is $\sim 10^{2}$ times as large as that in an ordinary electron-ion $(e-i)$ plasma, $\sim 2 m_{i} v_{\mathrm{A}}^{2}(M-1)$. Large-amplitude magnetosonic waves will propagate as shock waves.

\section{POSITRON MOTIONS}

In Sec. III A, we describe some properties of positron motions in an oblique shock wave. In Sec. III B, then, we will discuss positron acceleration. The shock wave is supposed to propagate in the $x$ direction with a propagation speed $v_{\text {sh }}$ in an external magnetic field $\mathbf{B}_{0}$ $=B_{0}(\cos \theta, 0, \sin \theta)$.

\section{A. Characteristics of positron motions}

The width of the transition region $\Delta$ of an oblique magnetosonic shock wave is of the order of the ion inertial length, $\sim c / \omega_{p i}$. The gyroradii of nonrelativistic positrons $\rho_{p}$ are thus much smaller than $\Delta$, while those of ions are comparable to or greater than $\Delta$. In addition, positrons can make gyromotion many times while they pass through the shock transition region. Hence, the motion of nonrelativistic positrons can be well described with drift approximation. Their guiding center velocity $\mathbf{v}_{\mathrm{g}}$ may thus be written as

$$
\mathbf{v}_{\mathrm{g}}=\mathbf{v}_{\mathrm{d}}+v_{\|} \frac{\mathbf{B}}{B},
$$

where $\mathbf{v}_{\mathrm{d}}$ is the drift velocity and $v_{\|}$is the velocity parallel to the magnetic field $\mathbf{B}$.

Another important property of positrons is that they can be reflected along the magnetic field by the parallel electric field $E_{\|}$. This occurs because the positron mass is quite small. (Ions can be reflected across the magnetic field. ${ }^{20,24,25}$ ) In fact, from the nonrelativistic equation of motion in the wave frame, we have the following equation: 


$$
\begin{aligned}
\frac{1}{2} m_{p}\left(v_{\mathrm{w} \|}-v_{\mathrm{rv}}\right)^{2}= & -e\left(F_{\mathrm{w}}-F_{\mathrm{w} 0}\right)-K-\mu_{\mathrm{w}} B_{\mathrm{w}}+\frac{1}{2} m_{p} v_{\mathrm{w} 0}^{2} \\
& -\frac{1}{2} m_{p} v_{\mathrm{wd}}^{2}-m_{p} c \frac{E_{\mathrm{w} y 0}}{B_{x 0}}\left(v_{\mathrm{wd} z}-v_{\mathrm{w} z 0}\right) .
\end{aligned}
$$

Here, the subscript $w$ refers to quantities in the wave frame, $v_{\mathrm{wd} z}$ is the $z$ component of the drift velocity $\mathbf{v}_{\mathrm{wd}}$, and $\mu_{\mathrm{w}}$ is the magnetic moment, $\mu_{\mathrm{w}}=m_{p} v_{\mathrm{w} \perp}^{2} /\left(2 B_{\mathrm{w}}\right)$ with $v_{\mathrm{w} \perp}$ the gyration speed perpendicular to the magnetic field. Also, $v_{\mathrm{rv}}$ $=-c E_{\mathrm{w} y 0} B_{\mathrm{w} z} /\left(B_{x 0} B_{\mathrm{w}}\right)$, and $K=-m_{p} v_{\mathrm{rv}}^{2} / 2$. The quantity $F_{\mathrm{w}}$ is the integral of $E_{\|}$along $\mathbf{B},{ }^{26}$

$$
F_{\mathrm{w}}=-\int \frac{\mathbf{E}_{\mathrm{w}} \cdot \mathbf{B}_{\mathrm{w}}}{B_{x 0}} \mathrm{~d} x_{\mathrm{w}} .
$$

Equation (13) indicates that positrons cannot penetrate regions where the values of the right-hand side become negative. Thus, if $e\left(F_{\mathrm{w}}-F_{\mathrm{w} 0}\right)$ becomes greater than the other terms, positrons will be reflected there. The other terms are of the order of $m_{p} v_{\mathrm{A}}^{2}$ or $m_{p} v_{\mathrm{T} p}^{2}\left(v_{\mathrm{T} p}\right.$ the thermal speed), whereas $e F$ in a shock wave can exceed $m_{e} c^{2}$ (see Ref. 26 or Sec. IV in the present paper). Hence, positron reflection along $\mathbf{B}$ can occur in a shock wave.

Reflected positrons would move with the shock wave for long periods of time if the condition

$$
v_{\mathrm{sh}} \sim c \cos \theta,
$$

is satisfied. Because time averaged particle velocity in the $x$ direction is given as

$$
\left\langle v_{x}\right\rangle \simeq v_{\|} \cos \theta,
$$

reflected positrons with $v \sim c$ would not be able to quickly run away ahead of the shock wave with condition (15).

\section{B. Motions of accelerated positrons}

We here study the acceleration of reflected positrons and obtain the energy increase rate for such particles. We discuss this in the laboratory frame.

We consider positrons moving with a shock wave; thus, their $v_{x}$ is

$$
v_{x} \simeq v_{\mathrm{sh}} .
$$

We assume that their speeds are very close to $c(\gamma \gg 1)$ and that

$$
v \frac{d \gamma}{d t} \gg \gamma\left|\frac{d \mathbf{v}}{d t}\right| .
$$

Also, we assume that $v_{y}$ is much smaller in magnitude than the other velocity components.

Hence, in the relativistic equation of motion,

$$
m_{p} \frac{d(\gamma \mathbf{v})}{d t}=e\left(\mathbf{E}+\frac{\mathbf{v}}{c} \times \mathbf{B}\right),
$$

we neglect $\gamma d v_{\sigma} / d t$ compared with $v_{\sigma} d \gamma / d t$, where $\sigma=x, y$, or $z$. Then, assuming that

$$
\begin{aligned}
& \frac{v_{\mathrm{sh}}}{c} \sim \frac{v_{z}}{c} \sim \frac{B_{x 0}}{B_{0}} \sim \frac{B_{z}}{B_{0}} \sim \frac{E_{y}}{B_{0}} \sim O(1), \\
& \frac{d \gamma}{d\left(\Omega_{p} t\right)} \sim \frac{v_{y}}{c} \sim \frac{B_{y}}{B_{0}} \sim \frac{E_{x}}{B_{0}} \sim \frac{E_{z}}{B_{0}} \sim O(\epsilon),
\end{aligned}
$$

where $\epsilon$ is a smallness parameter, we obtain the lowest order equations as

$$
\begin{aligned}
& m_{p} v_{\mathrm{sh}} \frac{d \gamma}{d t}=e E_{x}+e \frac{v_{y}}{c} B_{z}-e \frac{v_{z}}{c} B_{y}, \\
& 0=e \frac{v_{z}}{c} B_{x 0}-e \frac{v_{\mathrm{sh}}}{c} B_{z 0}, \\
& m_{p} v_{z} \frac{d \gamma}{d t}=-e \frac{v_{y}}{c} B_{x 0} .
\end{aligned}
$$

Here, we have used the relations

$$
E_{y}=\left(v_{\mathrm{sh}} / c\right)\left(B_{z}-B_{z 0}\right)
$$

and

$$
E_{z}=-\left(v_{\mathrm{sh}} / c\right) B_{y},
$$

which are obtained from Maxwell equations for a stationary wave, where the fields depend only on $\xi=x-v_{\text {sh }} t$. From Eq. (23), we find that these positrons move almost parallel to $\mathbf{B}_{0}$,

$$
v_{z} / v_{\mathrm{sh}}=B_{z 0} / B_{x 0} .
$$

Eliminating $v_{y}$ and $v_{z}$ in Eq. (22) with the aid of Eqs. (24) and (27), we find the time rate of change of $\gamma$ as

$$
\frac{d \gamma}{d t}=\frac{e B_{x 0}^{2}}{m_{p} v_{\mathrm{sh}}}\left(\frac{E_{x}(\xi)-v_{\mathrm{sh}} B_{z 0} B_{y}(\xi) /\left(c B_{x 0}\right)}{B_{z 0} B_{z}(\xi)+B_{x 0}^{2}}\right) .
$$

Here, the particle position $\xi$ and thus $\mathbf{E}(\xi)$ and $\mathbf{B}(\xi)$ are constant. The particle energy therefore increases linearly with time. The effect of the synchrotron radiation can be neglected in this mechanism if $\gamma^{2} B<10^{15}$, where $B$ is in Gauss (see the Appendix).

With the aid of Eqs. (25) and (26), we can put Eq. (28) into the form

$$
\frac{d \gamma}{d t}=\frac{e B_{x 0}}{m_{p} v_{\mathrm{sh}}} \frac{(\mathbf{E} \cdot \mathbf{B})}{\left(\mathbf{B} \cdot \mathbf{B}_{0}\right)} .
$$

We also note that the relation $\mathbf{E} \cdot \mathbf{B}=\mathbf{E} \cdot \mathbf{B}_{0}$ holds for stationary waves with Eqs. (25) and (26).

\section{SIMULATION STUDIES}

\section{A. Simulation method and parameters}

We now study the positron acceleration in a shock wave by means of a one-dimensional (one space coordinate and three velocity components), relativistic, electromagnetic particle simulation code with full particle dynamics. ${ }^{27}$ The method to initiate shock waves was described in detail in the preceding papers. ${ }^{26,28,29}$ That is, the particles near the left boundary of a simulation box initially have rightward momenta. They push the neighboring particles and excite a shock wave, which then evolves in a self-consistent manner. 


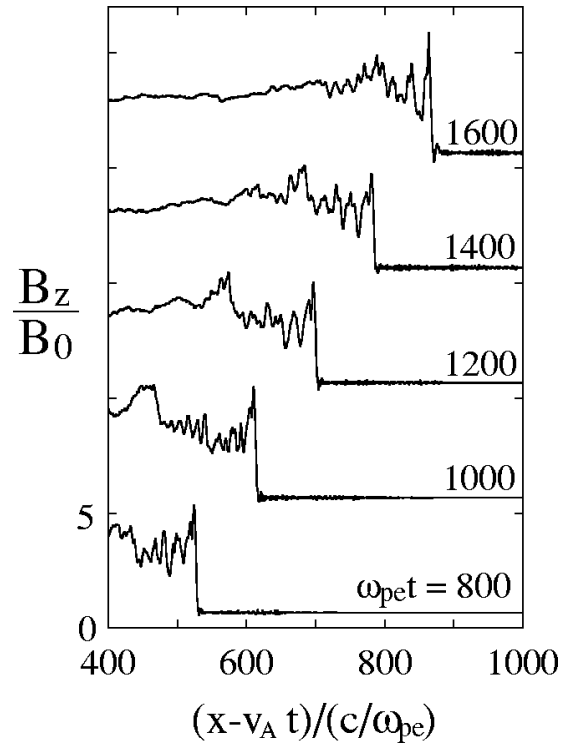

FIG. 2. Profiles of $B_{z}$ of an oblique shock wave at various times.

The simulation parameters are as follows. The total system length is $L_{x}=8192 \Delta_{\mathrm{g}}$, where $\Delta_{\mathrm{g}}$ is the grid spacing. The ion-to-electron mass ratio is $m_{i} / m_{e}=100$, with $m_{p}=m_{e}$. (These are rest masses.) The number of electrons is $N_{e}$ $=614,400$, with $N_{e}=N_{i}+N_{p}$. The initial thermal velocities are $v_{\mathrm{T} e} / c=v_{\mathrm{T} p} / c=0.08$ and $v_{\mathrm{T} i} / c=0.008$. The magnetic field strength is $\left|\Omega_{e}\right| / \omega_{p e}=3.0$ in the upstream region. The electron skin depth is $c / \omega_{p e}=4 \Delta_{\mathrm{g}}$. The time step is sufficiently small, $\omega_{p e} \Delta t=0.01$, so that $\Delta t$ is much smaller than the electron

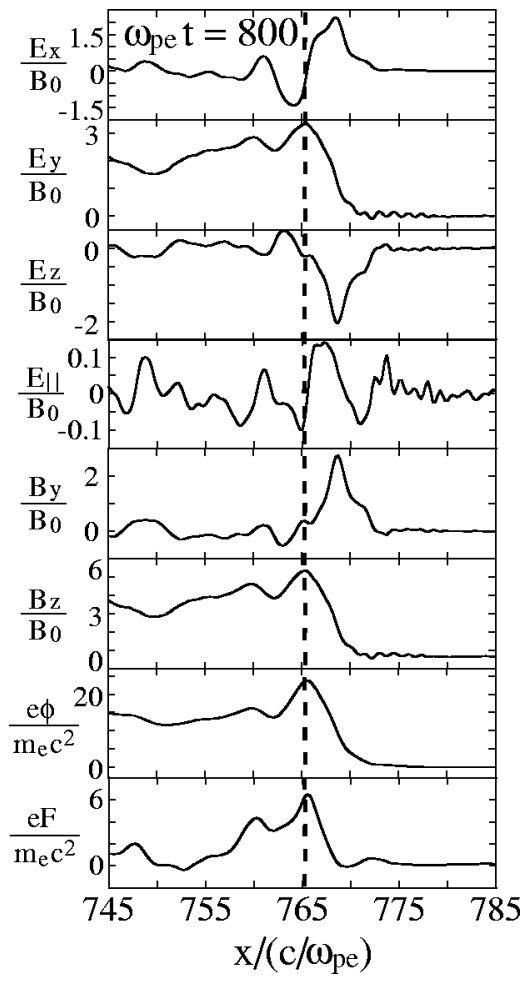

FIG. 3. Snapshots of field profiles. The vertical dashed line indicates the position at which $B_{z}$ has its maximum value.

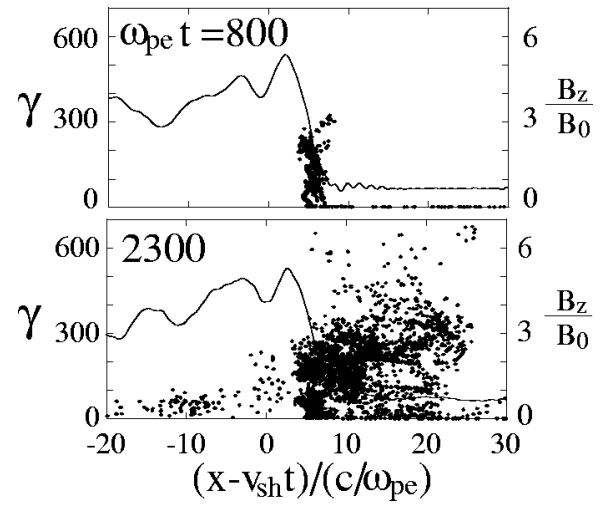

FIG. 4. Phase space plots $(x, \gamma)$ of positrons. Profiles of $B_{z}$ are also shown.

gyroperiod even in the shock region. As in the theoretical model, the external magnetic field is in the $(x, z)$ plane, and waves propagate in the $x$ direction.

\section{B. Simulation result}

Figure 2 shows profiles of $B_{z}$ in a shock wave at various times. Here, the shock propagation angle is $\theta=42^{\circ}$. The positron density is taken to be $n_{p 0} / n_{e 0}=0.02$; thus, $v_{\mathrm{A}} / c$ $=0.301$. The shock speed is observed to be $v_{\mathrm{sh}}=2.42 v_{\mathrm{A}}$; hence, $v_{\mathrm{sh}} \sim c \cos \theta$ is satisfied. The main pulse propagates nearly steadily. Figure 3 displays field profiles at $\omega_{p e} t=800$. The fields $E_{y}, B_{z}, \phi$, and $F$ have similar profiles. The maximum value of $F$ is much greater than $m_{p} v_{\mathrm{A}}^{2}$, suggesting that positrons can be reflected along $\mathbf{B}$.

We show in Fig. 4 phase space plots $(x, \gamma)$ of positrons and profiles of $B_{z}$. Here, some positrons are accelerated up to $\gamma \sim 700$. In the shock transition region, positrons are reflected by the large positive $F$; in the top panel, we find no positrons behind the shock transition region. As these reflected positrons move with the shock wave, they gain ener-

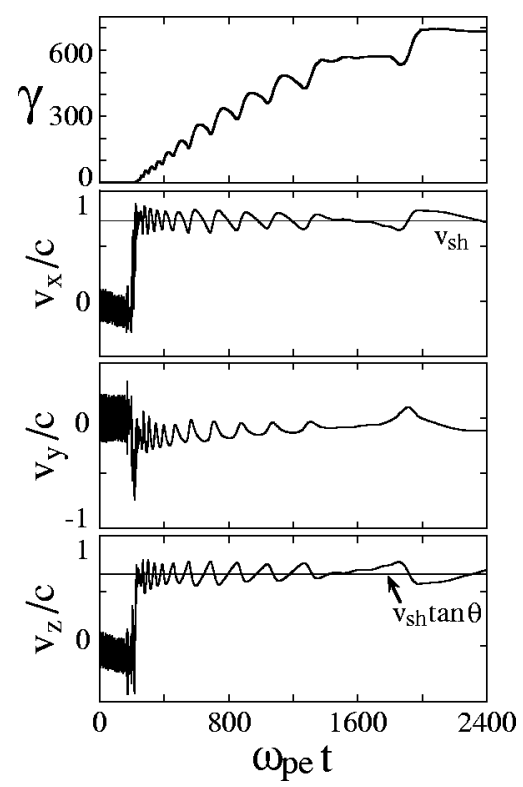

FIG. 5. Time variations of $\gamma, v_{x}, v_{y}$, and $v_{z}$ of an accelerated positron. 


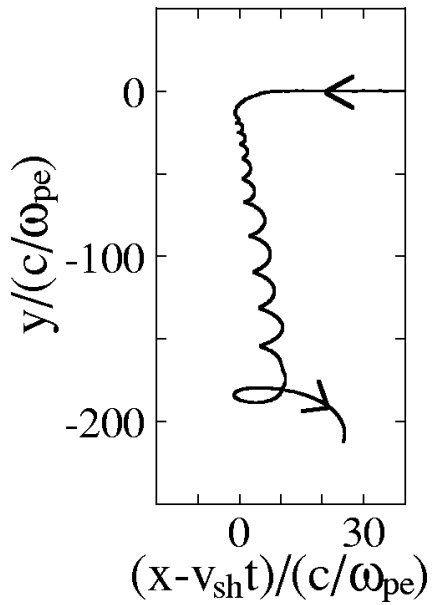

FIG. 6. Orbit of an accelerated positron in the $\left(x-v_{\mathrm{sh}} t, y\right)$ plane.

gies from the wave. (In the upstream region, positrons have a Maxwellian velocity distribution with a thermal velocity much lower than the speed of light.)

Figure 5 shows time variations of $\gamma$ and $\mathbf{v}$ of a positron accelerated to $\gamma \sim 700$. After the encounter with the shock wave at $\omega_{p e} t \simeq 250$, this particle was reflected. Its $\gamma$ linearly increased with time from $\omega_{p e} t \simeq 250$ to $\omega_{p e} t \simeq 1600$. The energy increase rate is $d \gamma / d\left(\omega_{p e} t\right)=0.41$, which is close to the theoretical value $d \gamma / d\left(\omega_{p e} t\right)=0.38$ estimated from Eq. (28). Here, in the theoretical estimate, we have used simulation data for the field values; $\left\langle E_{x} / B_{0}\right\rangle=1.14,\left\langle B_{y} / B_{0}\right\rangle=1.18$, and $\left\langle B_{z} / B_{0}\right\rangle=2.45$, where the brackets denote time averaging.

The thin horizontal lines in the panels of $v_{x}$ and $v_{z}$ represent the velocities $v_{x}=v_{\mathrm{sh}}$ and $v_{z}=v_{\mathrm{sh}} \tan \theta$, respectively. These pictures indicate that $v_{x} \approx v_{\text {sh }}$ on average and that the particle moves nearly parallel to the external magnetic field. From the third panel, we find that $v_{y}$ is negative and is small in magnitude for $\omega_{p e} t \lesssim 1800$. These are consistent with the theory.

Figure 6 shows the trajectory of this positron in the $(x$ $\left.-v_{\mathrm{sh}} t, y\right)$ plane. After the reflection, this particle moves in the negative $y$ direction along the shock front with hypotrochoidlike orbit. In the final stage, however, the trajectory changes

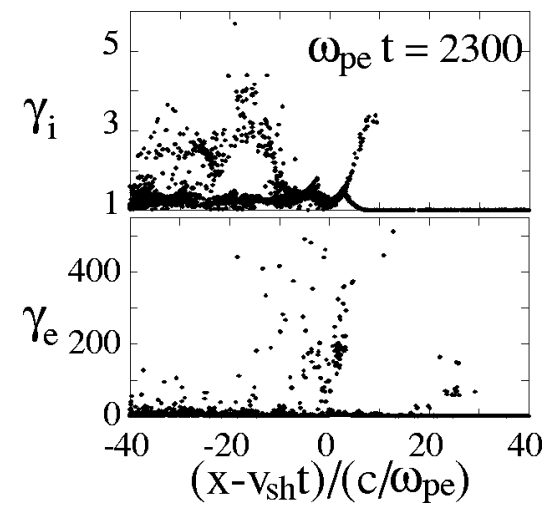

FIG. 7. Phase space plots $(x, \gamma)$ of ions and electrons.

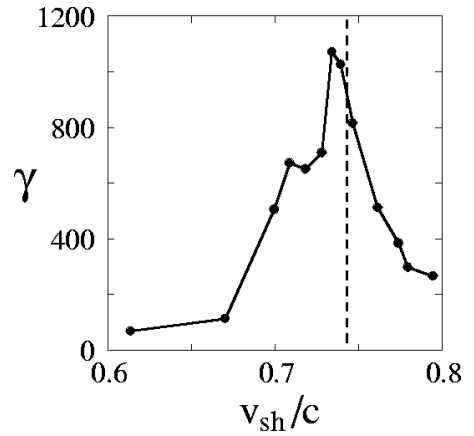

FIG. 8. The maximum $\gamma$ vs shock speed $v_{\text {sh }}$. The other parameters are the same as those in Fig. 2.

to epitrochoidlike orbit; at this stage, $\gamma$ shows a stepwise increase as in the incessant acceleration; ${ }^{30-33}$ in Fig. 5, $\gamma$ jumped at $\omega_{p e} t \simeq 1900$.

Ions and electrons are also accelerated by this shock wave with other different mechanisms; for the ion and electron acceleration mechanisms, see Refs. 24, 28, and 34-37 and Refs. 26, 38, and 39, respectively. Figure 7 shows phase space plots of ions (the upper panel) and of electrons (the lower panel). Some ions are reflected across the magnetic field and are accelerated to $\gamma \sim 5$. On the other hand, some electrons gained ultrarelativistic energies, $\gamma \sim 500$.

Next, we investigate parameter dependence of the positron acceleration. Figure 8 shows the maximum value of $\gamma$ as a function of the shock speed $v_{\mathrm{sh}}$. The other parameters are the same as those in the above simulation. Here, the dashed vertical line represents the shock speed $v_{\mathrm{sh}}=c \cos \theta$. For a fairly wide range of $v_{\text {sh }}, \gamma$ exceeds 100 . In particular, positron energies become very high, $\gamma \sim 1000$, when $v_{\text {sh }}$ $\sim c \cos \theta$.

Figure 9 shows the maximum value of $\gamma$ as a function of the propagation angle $\theta$. The propagation speeds for these shocks were fixed to be $v_{\mathrm{sh}} / c \simeq 0.73$; the other parameters were unchanged. For these parameters, $\gamma$ exceeds 100 for $30^{\circ} \lesssim \theta \lesssim 50^{\circ}$. Similarly to Fig. 8, $\gamma$ has a peak near the angle satisfying the relation $v_{\mathrm{sh}}=c \cos \theta$; this angle is indicated by the dashed vertical line.

Figure 10 shows the maximum value of $\gamma$ as a function of the mass ratio $m_{i} / m_{e}$. Here, $v_{\mathrm{A}}, v_{\mathrm{sh}}$, and $\theta$ were fixed to be the same as those in Fig. $2 ; v_{\mathrm{A}} / c=0.301, v_{\mathrm{sh}} / c \simeq 0.73$, and

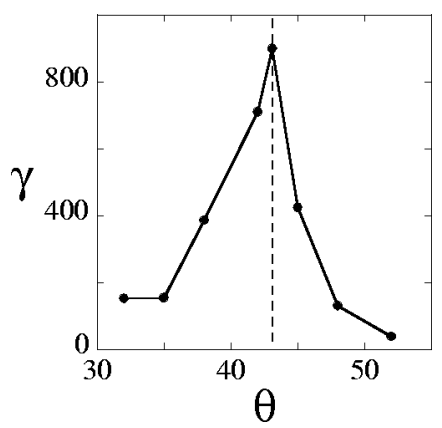

FIG. 9. The maximum $\gamma$ vs propagation angle $\theta$. The propagation speeds for these shocks are $v_{\mathrm{sh}} / c \simeq 0.73$. 


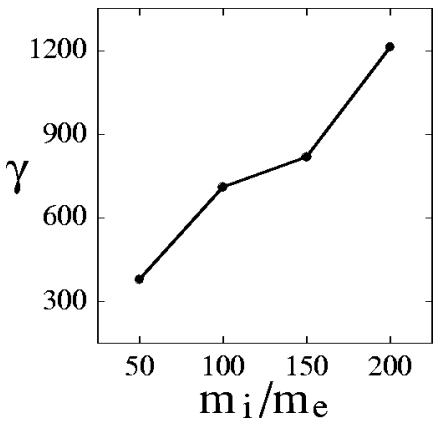

FIG. 10. The maximum $\gamma$ vs mass ratio $m_{i} / m_{e}$. The propagation angle $\theta$ and Alfvén speed $v_{\mathrm{A}}$ are fixed to be $\theta=42^{\circ}$ and $v_{\mathrm{A}} / c=0.301$.

$\theta=42^{\circ}$. The thermal velocities were also unchanged. Under these conditions, the maximum value of $\gamma$ increases almost linearly with $m_{i} / m_{e}$.

When the positron density is high, $n_{p 0} \sim n_{i 0}$, this acceleration mechanism is less effective. Figure 11 displays positron phase space plots $(x, \gamma)$ at $\omega_{p e} t=2100$ for $n_{p 0} / n_{e 0}$ $=0.02,0.1$, and 0.5. Again, $v_{\mathrm{A}}, v_{\mathrm{sh}}$, and $\theta$ are the same as those in Fig. 2. For $n_{p 0} / n_{e 0}=0.5$, the fraction of accelerated positrons is small, and their maximum energy is rather low, $\gamma \lesssim 300$. (The acceleration to $\gamma \gtrsim 100$ in this case is caused by the interaction between $E_{y}$ and gyromotion perpendicular to $\mathbf{B} .^{30-33}$ ) That the acceleration along $\mathbf{B}$ is weak is due to the fact that, as $n_{p 0}$ rises, the quantity $F$ becomes small and non-stationary; Fig. 12 compares profiles of $F$ for three different values of $n_{p 0} / n_{e 0}$.

\section{SUMMARY}

We have studied positron acceleration in an oblique magnetosonic shock wave in an $e-p-i$ plasma. Nonlinear oblique magnetosonic pulses propagate with pulse width $\Delta$ $\sim c / \omega_{p i}$ and electric potential $e \phi \gtrsim 2 m_{i} v_{\mathrm{A}}^{2}(M-1)$. From the relativistic equation of motion, then, we have found that positrons can be accelerated in the shock transition region, mov-

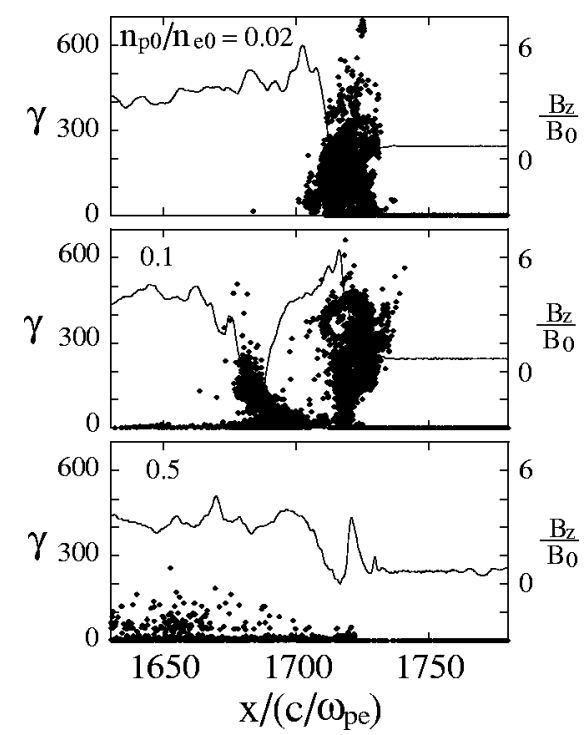

FIG. 11. Positron phase space plots $(x, \gamma)$ for $n_{p 0} / n_{e 0}=0.02,0.1$, and 0.5 . Here, $v_{\mathrm{A}} / c=0.301, v_{\mathrm{sh}} / c \simeq 0.73$, and $\theta=42^{\circ}$.

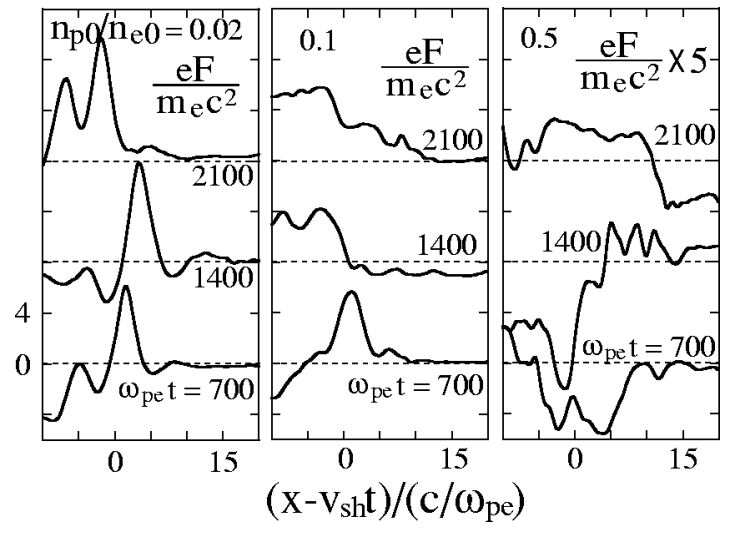

FIG. 12. Profiles of $F$ for three different positron densities. The values of $F$ for $n_{p 0} / n_{e 0}=0.5$ are magnified five times.

ing nearly parallel to the external magnetic field. The energy increase rate is proportional to $\mathbf{E} \cdot \mathbf{B}$. Then, we have demonstrated this acceleration with a one-dimensional, relativistic, electromagnetic particle simulation code with full particle dynamics. It has been shown that an oblique shock wave reflect some positrons and then accelerate them to ultrarelativistic energies with this mechanism; positrons with $\gamma$ $\sim 1000$ have been observed. Further, we have examined the parameter dependence of this acceleration. For fairly wide ranges of $v_{\text {sh }}$ and $\theta$, positrons are accelerated to energies $\gamma$ $\gtrsim 100$; the acceleration is particularly enhanced when $v_{\mathrm{sh}}$ $\sim c \cos \theta$. Also, the acceleration is strong when the positron density is rather low, $n_{p 0} / n_{e 0} \lesssim 0.1$.

\section{ACKNOWLEDGMENTS}

This work was carried out by the joint research program of the National Institute for Fusion Science and was supported in part by a Grant-in-Aid for Scientific Research from the Japan Society for the Promotion of Science.

\section{APPENDIX: COMPARISON BETWEEN THE ENERGY INCREASE RATE AND RADIATION LOSS RATE}

The energy loss rate by the synchrotron radiation from a particle with velocity perpendicular to the magnetic field, $\mathbf{v}$ $=\mathbf{v}_{\perp}$, is given by ${ }^{40}$

$$
-\frac{d \gamma}{d\left(\Omega_{p} t\right)_{\mathrm{syn}}}=\frac{2 e^{2} \Omega_{p} \gamma^{2} v_{\perp}^{2}}{3 m_{p} c^{5}}=1.1 \times 10^{-16} \times \frac{v_{\perp}^{2}}{c^{2}} \gamma^{2} B_{(\mathrm{G})},
$$

where $B_{(\mathrm{G})}$ is the magnetic field strength in Gauss. The synchrotron radiation from a positron accelerated to energy $\gamma$ by the present mechanism will be much smaller than Eq. (A1), because the particle velocity is nearly parallel to $\mathbf{B}_{0}$. On the other hand, from Eqs. (24) and (27), we may write the energy increase rate of the accelerated positron as

$$
\frac{d \gamma}{d\left(\Omega_{p} t\right)_{\mathrm{acc}}}=\frac{\left(-v_{y}\right) \cos \theta}{v_{\mathrm{sh}} \tan \theta} .
$$

Their ratio is 


$$
\frac{-d \gamma / d\left(\Omega_{p} t\right)_{\mathrm{syn}}}{d \gamma / d\left(\Omega_{p} t\right)_{\mathrm{acc}}}=\frac{2}{3} \frac{\left[e^{2} /\left(m_{p} c^{2}\right)\right]}{\left(c / \Omega_{p}\right)} \frac{v_{\perp}^{2} v_{\mathrm{sh}} \tan \theta}{c^{2}\left(-v_{y}\right) \cos \theta} \gamma^{2} .
$$

Assuming that $v_{\perp} \sim c, v_{y} \sim-0.1 c, v_{\mathrm{sh}} \sim c \cos \theta$, and $\tan \theta$ $\sim 1$, we have

$$
\frac{-d \gamma / d\left(\Omega_{p} t\right)_{\mathrm{syn}}}{d \gamma / d\left(\Omega_{p} t\right)_{\mathrm{acc}}} \sim 10^{-15} \times \gamma^{2} B_{(\mathrm{G})} .
$$

We can neglect the effect of the synchrotron radiation if $\gamma^{2} B_{(\mathrm{G})}<10^{15}$.

${ }^{1}$ T. E. Cowan, M. D. Perry, M. H. Key et al., Laser Part. Beams 17, 773 (1999).

${ }^{2}$ P. A. Sturrock, Astrophys. J. 164, 529 (1971).

${ }^{3}$ C. F. Kennel and F. V. Coroniti, Astrophys. J. 283, 694 (1984).

${ }^{4}$ C. S. Reynolds, A. C. Fabian, A. Celotti, and M. J. Rees, Mon. Not. R. Astron. Soc. 283, 873 (1996).

${ }^{5}$ K. Hirotani, S. Iguchi, M. Kimura, and K. Wajima, Publ. Astron. Soc. Jpn. 51, 263 (1999).

${ }^{6}$ S. Coutu, S. W. Barwick, J. J. Beatty et al., Astropart. Phys. 11, 429 (1999).

${ }^{7}$ C. Grimani, S. A. Stephens, F. S. Cafagna et al., Astron. Astrophys. 392, 287 (2002).

${ }^{8}$ C. F. Kennel and R. Pellat, J. Plasma Phys. 15, 335 (1976).

${ }^{9}$ J.-I. Sakai and T. Kawata, J. Phys. Soc. Jpn. 49, 753 (1980).

${ }^{10}$ M. Ashour-Abdalla, J. N. Leboeuf, T. Tajima, J. M. Dawson, and C. F. Kennel, Phys. Rev. A 23, 1906 (1981).

${ }^{11}$ M. Hoshino, J. Arons, Y. A. Gallant, and A. B. Langdon, Astrophys. J. 390, 454 (1992).

${ }^{12}$ G. A. Stewart and E. W. Laing, J. Plasma Phys. 47, 295 (1992).

${ }^{13}$ G. S. Lakhina and F. Verheest, Astrophys. Space Sci. 253, 97 (1997).

${ }^{14}$ V. I. Berezhiani and S. M. Mahajan, Phys. Rev. Lett. 73, 1110 (1994).
${ }^{15}$ C. Joshi, B. Blue, E. Clayton et al., Phys. Plasmas 9, 1845 (2002).

${ }^{16}$ B. E. Blue, C. E. Clayton, C. L. O'Connell et al., Phys. Rev. Lett. 90, 214801 (2003).

${ }^{17}$ H. Hasegawa, S. Irie, S. Usami, and Y. Ohsawa, Phys. Plasmas 9, 2549 (2002).

${ }^{18}$ H. Hasegawa and Y. Ohsawa, J. Phys. Soc. Jpn. 73, 1764 (2004).

${ }^{19}$ H. Hasegawa, S. Usami, and Y. Ohsawa, Phys. Plasmas 10, 3455 (2003).

${ }^{20}$ R. Z. Sagdeev and V. D. Shapiro, Zh. Eksp. Teor. Fiz. Pis'ma Red. 17, 387 (1973) [JETP Lett. 17, 279 (1973)].

${ }^{21}$ M. A. Lee, V. D. Shapiro, and R. Z. Sagdeev, J. Geophys. Res., [Oceans] 101, 4777 (1996)

${ }^{22}$ T. Katsouleas and J. M. Dawson, Phys. Rev. Lett. 51, 392 (1983).

${ }^{23}$ R. Sugihara and Y. Midzuno, J. Phys. Soc. Jpn. 47, 1290 (1979).

${ }^{24}$ Y. Ohsawa, J. Phys. Soc. Jpn. 59, 2782 (1990).

${ }^{25}$ T. Kawashima, S. Miyahara, and Y. Ohsawa, J. Phys. Soc. Jpn. 72, 1664 (2003).

${ }^{26}$ N. Bessho and Y. Ohsawa, Phys. Plasmas 6, 3076 (1999).

${ }^{27}$ P. C. Liewer, A. T. Lin, J. M. Dawson, and M. Z. Caponi, Phys. Fluids 24, 1364 (1981).

${ }^{28}$ Y. Ohsawa, Phys. Fluids 28, 2130 (1985).

${ }^{29}$ M. Toida and Y. Ohsawa, Sol. Phys. 171, 161 (1997).

${ }^{30}$ K. Maruyama, N. Bessho, and Y. Ohsawa, Phys. Plasmas 5, 3257 (1998).

${ }^{31}$ T. Masaki, H. Hasegawa, and Y. Ohsawa, Phys. Plasmas 7, 529 (2000).

${ }^{32}$ S. Usami, H. Hasegawa, and Y. Ohsawa, Phys. Plasmas 8, 2666 (2001).

${ }^{33}$ S. Usami and Y. Ohsawa, Phys. Plasmas 9, 1069 (2002).

${ }^{34}$ D. Biskamp and H. Welter, Nucl. Fusion 12, 663 (1972).

${ }^{35}$ D. W. Forslund, K. B. Quest, J. U. Brackbill, and K. Lee, J. Geophys. Res., [Oceans] 89, 2142 (1984).

${ }^{36}$ R. L. Tokar, S. P. Gary, and K. B. Quest, Phys. Fluids 30, 2569 (1987).

${ }^{37}$ B. Lembege and J. M. Dawson, Phys. Fluids B 1, 1001 (1989).

${ }^{38}$ N. Bessho and Y. Ohsawa, Phys. Plasmas 7, 4004 (2000).

${ }^{39}$ N. Bessho and Y. Ohsawa, Phys. Plasmas 9, 979 (2002).

${ }^{40}$ L. D. Landau and E. M. Lifshitz, The Classical Theory of Fields (Pergamon, New York, 1975). 